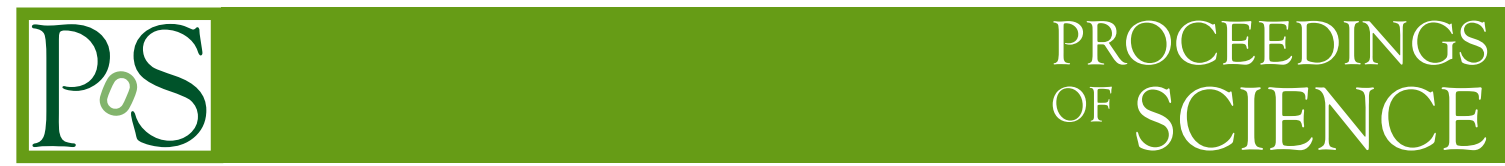

\title{
ATLAS L1 track trigger for super-LHC
}

\author{
Nikos Konstantinidis* (on behalf of the ATLAS collaboration) \\ Department of Physics and Astronomy, University College London, London \\ E-mail: Nikolaos.Konstantinidisecern.ch
}

I present preliminary ideas and feasibility studies of a L1 track trigger for the ATLAS detector upgrade for super-LHC. These studies so far indicate that a design based on the selective readout of regions of interest in the tracker, driven by the L1 Muon and Calorimeter information, is best suited for ATLAS. We describe the details of this approach, its advantages and the implications for the ATLAS upgrade programme.

VERTEX 2009 (18th workshop) - VERTEX 2009

September 13 - 182009

Veluwe, the Netherlands

${ }^{*}$ Speaker. 


\section{Trigger/DAQ challenges at $S L H C$ and motivation for an $L 1$ track trigger}

As the LHC is about to record it's first collisions, plans and R\&D are already underway to upgrade the detectors and the accelerator, so that in about a decade the peak instantaneous luminosity of the super-LHC (sLHC) will reach $10^{35} \mathrm{~cm}^{-2} \mathrm{~s}^{-1}$, an order of magnitude higher than the design luminosity of the LHC.

Depending on the design options for the accelerator upgrade currently under consideration [1], there may be up to 400 proton-proton collisions per bunch crossing. These will pose formidable challenges to the LHC detectors. In particular, the two main challenges for the ATLAS Trigger/DAQ system are:

- The much higher particle densities will lead to a corresponding increase of the hit occupancy in the detectors. Therefore, even for the same L1-Accept (L1A) rate of $100 \mathrm{kHz}$ as in the current system, a much larger data volume will need to be readout from the detector, hence a significant increase of the readout bandwidth will be necessary. The same argument is true for the rate of storage to disk. Even at the current rate of a few hundred $\mathrm{Hz}$, the recorded data volume will be a factor between 5 and 10 higher;

- To first order, the trigger rates will go up by an order of magnitude compared to $10^{34} \mathrm{~cm}^{-2} \mathrm{~s}^{-1}$, with the same $p_{T}$-thresholds. At the same time pattern recognition and feature extraction will be much more challenging in these very busy events. Therefore, in order to avoid having to raise the L1 $p_{T}$-thresholds to values that may compromise the physics potential of ATLAS, the L1 trigger will need to borrow as much as possible algorithms and techniques that are currently used in the High level Triggers (HLT). On the other hand, the HLT selection will inevitably have to become more exclusive. For instance, the cross section of $W$ boson production is such that the rate of $W \rightarrow e / \mu v$ at $10^{35} \mathrm{~cm}^{-2} \mathrm{~s}^{-1}$ is more than a $\mathrm{kHz}$, hence it will not be possible to simply record all events with a single, isolated electron or muon.

Given the above challenges, the ATLAS Trigger system at sLHC has to be designed to be robust and flexible, so that it can be adjusted to exploit the full physics potential of sLHC. For the L1 Trigger, in particular, it is essential to try and maximize the purity of the physics events that are read out from the detector, making in this way the best use of the available bandwidth.

Apart from possible upgrades to the existing L1 Calorimeter and Muon trigger systems, the only other source of information that has the potential to enhance the purity of the events selected at L1 is the Inner Detector. Given that a new tracker will be built, there is the opportunity to instrument it with L1 triggering capabilities. Indeed, in the current ATLAS, where tracking is not used at L1, tracking information is an essential ingredient in the event selection at the HLT. Matching tracks found in the Inner Detector with objects in the calorimeters or the muon detector is a key handle for achieving the required background rejection at the current L2 Trigger. In addition to this matching, tracking information would have two more important uses for the L1 Trigger at sLHC. First, it would allow verification of whether multiple L1 trigger objects came from the same proton-proton interaction. This would significantly reduce the rate of double lepton triggers, which is expected to be dominated by events where two objects (most often a true lepton and a fake L1 lepton) come from different $z$ positions along the beam line. Second, it may provide the capability to perform 
track-based isolation for L1 electron, muon or tau candidates, again based on the $z$-positions of tracks along the beam line. Finally, a track trigger would provide much needed flexibility and redundancy, and therefore robustness, to the L1 Trigger system. This is vital, especially given that the detailed physics goals of the sLHC will only be known after some years of running at the LHC. This flexibility should ensure that the full physics potential of the sLHC can be exploited, irrespective of what the LHC discovers.

\section{ATLAS L1 Trigger upgrade plans}

The L1 Track Trigger (L1Track) studies are part of the overall studies for the upgrade of the ATLAS L1 Trigger for sLHC. In order to put the L1 Track Trigger studies into context, a brief description of the overall L1 Trigger upgrade plans is given here.

The L1 Trigger system is expected to be completely redesigned for sLHC. The current system [2] comprises the L1 Calorimeter Trigger (L1Calo), the L1 Muon Trigger (L1Muon) and the Central Trigger Processor (CTP).

The current L1Calo system uses Trigger towers of granularity $0.1 \times 0.1$ in $(\eta, \phi)$. The ATLAS Calorimeters have much finer granularity and the ATLAS Calorimeter communities plan to upgrade the readout systems of the Calorimeters, so that signals are digitized on-detector and readout at $40 \mathrm{MHz}$. Therefore, the upgraded L1Calo system will potentially have access to the full Calorimeter granularity. Studies are underway to determine what level of granularity will be necessary or, indeed, possible to use within the constraints of L1, from the current L2 Calorimeter Trigger it is clear that the longitudinal segmentation of the Liquid Argon (LAr) electromagnetic calorimeter, as well as the fine segmentation in eta of the first LAr sampling ("strips" of $0.003 \times 0.1$ in $\eta, \phi$ ) will be significant in rejecting $\pi^{0}$ 's and photon conversions. This is an important difference between ATLAS and CMS, with possible implications for the overall design of the upgraded L1 Trigger systems of the two experiments.

The L1Muon performance can be improved by improving its $p_{T}$ resolution. As can be seen in Fig. 1, even ignoring cavern background and pattern recognition issues, the L1Muon rate for higher $p_{T}$-thresholds will be dominated by true muons of lower $p_{T}$, and will exceed the $100(/ 50) \mathrm{kHz}$ for a $20(/ 40) \mathrm{GeV} p_{T}$-threshold at $10^{35} \mathrm{~cm}^{-2} \mathrm{~s}^{-1}$. Studies are currently underway to investigate if the addition of more precise planes of Muon chambers can provide better muon $p_{T}$ resolution.

Finally, the initial plans for the CTP are that it will give its place to a Global Trigger Processor, that will receive from the $\mathrm{L} 1 \mathrm{Calo} / \mathrm{Muon}$ (and Track) systems a set Trigger objects, each containing features, such as $\left(\eta, \phi, E_{T}\right)$, like the C++ objects in the current HLT, and will combine these object to make the global decision, using similar ideas to the current HLT.

\section{The regional readout approach for the L1 Track Trigger}

Given the colossal number of channels in the Inner Detector, it is not feasible to read out the entire detector at $40 \mathrm{MHz}$ to produce L1Track information. Two different approaches for reducing the volume of tracker data to be read out for L1Track are currently under consideration in ATLAS. The first is a regional readout of the Inner Detector, based on Region-of-Interest (RoI) information from L1Calo and L1Muon; the second is a standalone approach, using dedicated tracker layers to 


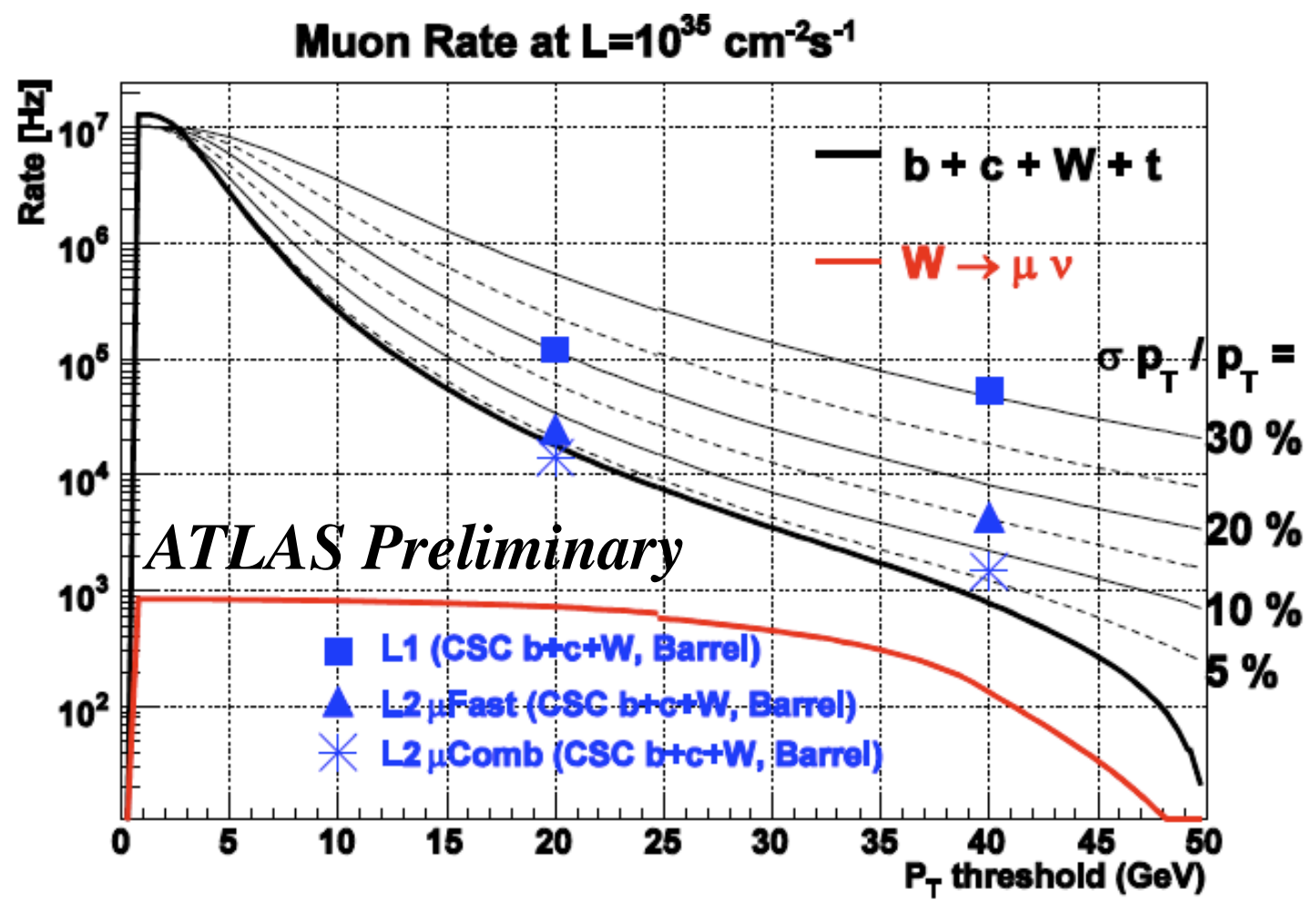

Figure 1: The rate of muons at $10^{35} \mathrm{~cm}^{-2} \mathrm{~s}^{-1}$ as a function of the muon $p_{T}$, estimated by a simple convolution of the physics cross section with various $p_{T}$ resolutions. The symbols show the performance of the current Muon Triggers at $20 \mathrm{GeV}$ and $40 \mathrm{GeV}$ : squares, for the L1Muon performance, triangles for the L2 Muon software algorithm (L2 $\mu$ Fast), and asterisks for the combined L2 Muon+Inner Tracker algorithm (L2 $\mu$ Comb).

select hits from high- $p_{T}$ tracks and read out only those for further processing. The latter approach is pursued as the baseline by CMS [3]. The former is receiving more attention in ATLAS and will be discussed more here.

The regional readout approach assumes that L1Calo and L1Muon would reduce the rate from $40 \mathrm{MHz}$ to (order of) $500 \mathrm{kHz}$. At this rate, they identify Regions of Interest (RoIs) in $(\eta, \phi)$ and send fast readout requests only to tracker modules that are inside an RoI. In this way, only a small fraction of the tracker would be read out, and only at a reduced rate, so the required additional bandwidth for the Inner Detector would be very modest. Several variations are possible in this approach, depending on how fast the regional Inner Detector data can be read out and processed, and on the overall L1 Trigger latency envelope. Ideally, tracking information should be used directly within the L1 Trigger. However, an option for a two-stage L1 trigger is also under consideration, for use if the Inner Detector readout is too slow. This would require additional buffers for all ATLAS sub-detectors, in which data would be held after a first-step decision (for instance, the one based only on L1Muon and L1Calo) until the slower, definitive L1 Trigger decision was available.

While a standalone track trigger is attractive, CMS studies ([3]) have shown that for it to be robust and achieve the required performance, such a system would be very complex and would 

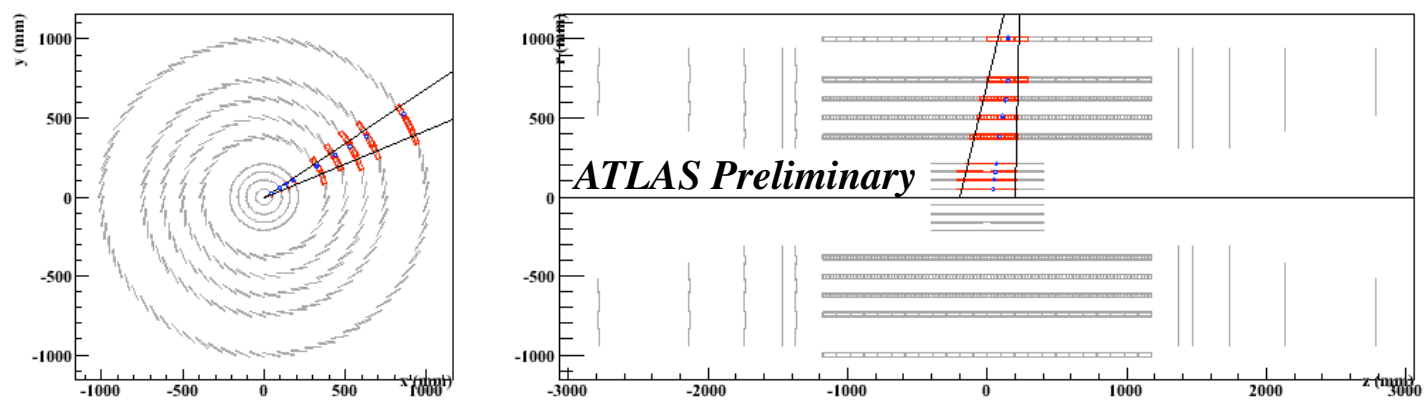

Figure 2: A lepton RoI in the tracker, in the $x-y$ and $\rho-z$ projections. The RoI is wider in $z$ close to the beam line to account for the spread of the luminous region in $z$.

introduce significantly more material in the tracker than otherwise. For ATLAS, the prospects for improvements in L1Calo and L1Muon, and in particular the potential of rejecting effectively $\pi^{0}$ 's and photon conversions with the use of the LAr Calorimeter first sampling, suggest that such a complex L1Track system may not be necessary. Indeed, the regional readout approach has no impact on the layout of the tracker - this can be optimized for offline tracking and for minimal material - and the additional material introduced can indeed be rather small, almost negligible.

A typical lepton RoI in the tracker is shown in Fig. 2. On average, such an RoI contains about $1 \%$ of the tracker modules and at $500 \mathrm{kHz}$ a small number of RoIs are expected, meaning that less than $10 \%$ of the tracker data will have to be read out at this rate.

Studies are currently underway to evaluate the latency requirements, dead time issues, the mechanism for the broadcast of the regional readout request to the targeted modules etc. As mentioned above, there are two variants of the regional readout approach:

- Longer pipelines: After the initial selection (which brings the rate down from $40 \mathrm{MHz}$ to $\sim 500 \mathrm{kHz}$ ), L1Muon and L1Calo broadcast regional readout requests only to the tracker modules that are inside RoIs. The regional tracker data are transmitted to the L1Track processors, while the data in all the ATLAS sub-detectors (including the tracker) stay in the L1 pipelines until the global decision, including tracking information, is produced. All the other sub-detectors see only this global decision - like the current L1A. With the use of tracking information, the full detector readout can be less than the nominal $100 \mathrm{kHz}$ (possibly as low as $50 \mathrm{kHz}$ or so), hence the overall effective readout rate for the tracker, including the regional data, can be within $100 \mathrm{kHz}$. The current estimates suggest that the overall latency of the above system will be close to $10 \mu \mathrm{s}$. This means that all ATLAS front-end electronics will have to be replaced. This approach would lead to a completely synchronous L1 system.

- Smart buffers in the front-end electronics: After the initial L1Calo/L1Muon selection, a Level-0-Accept (L0A) signal is broadcasted to the entire detector. The tracker data move from the L1 pipelines to "smart buffers" in the front-end ASICs. The regional readout requests are propagated to the tracker modules inside RoIs and the regional data are sent to the L1Track processors (as before). Other sub-detectors can do the same as the tracker (i.e. use smart buffers) or use the L0A signal as the current L1A and perform full readout at the L0A 
rate, if that is easier. The global Trigger decision, may come significantly later $(\sim 100 \mu \mathrm{s})$, depending on the capacity of the smart buffers and the LOA rate. Given that a lot more time will be available for the global decision, the full detector readout rate can end up being significantly lower than $100 \mathrm{kHz}$ (possibly $20-50 \mathrm{kHz}$ ). The advantage of this approach is that it relaxes the latency and synchronisity constraints and gives potentially more flexibility in terms of what Trigger processing can be done in tens of microseconds.

From the above discussion, it becomes clear that the regional readout approach for L1Track will have significant implications for the readout architecture of all the ATLAS sub-detectors. However, this two-step approach for the L1 decision has stimulated quite a lot of interest within the upgrade communities of the various ATLAS sub-detectors. With a two-step decision before the full detector readout, it will be possible to bring in to the L1 decision some more slower detectors, as, for example, the precise Monitored Drift Tubes (MDTs) in the Muon system, which can help to sharpen the $p_{T}$-threshold of the L1Muon system.

\section{Conclusions and outlook}

Many feasibility studies and qualitative arguments motivate the need for tracking information in the L1 Trigger of ATLAS at sLHC. Based on the studies so far, the regional readout approach appears to be best suited for ATLAS at sLHC, as it can provide sufficient tracking information at the L1 Trigger, without compromising the performance of the rest of ATLAS. The track trigger could prove to be a vital component in the design of a robust and flexible Trigger system that will be able to cope with the challenging conditions at sLHC and maximize the physics reach of ATLAS.

Simulation studies with large, fully simulated data sets are about to start in order to develop a robust physics case for L1Track. Results should become available within the next six months or so, in time for the ATLAS Upgrade Letter of Intent. Following these studies, the detailed design of the L1Track system will be developed.

\section{Acknowledgments}

I wish to thank the organisers for inviting me to give this talk and for the very interesting (scientific and social!) programme of the workshop. I would also like to thank my ATLAS TDAQ colleagues, especially M. Warren, G. Crone, R. Brenner, E. Lipeles and K. Nagano for useful discussions and contributions to the ATLAS L1 track trigger project.

\section{References}

[1] Frank Zimmermann, "SLHC machine plans", talk given at the ATLAS Upgrade Week, February 2009, http://indico.cern.ch/conferenceDisplay.py?confId=45460\#2009-02-27.

[2] G. Aad et al. [ATLAS Collaboration], "The ATLAS Experiment at the CERN Large Hadron Collider", JINST 3 S08003 (2008).

[3] Anders Ryd, "CMS L1 Track Trigger", these proceedings 